\title{
Characteristic Acylcarnitine Profiles in Inherited Defects of Peroxisome Biogenesis: A Novel Tool for Screening Diagnosis Using Tandem Mass Spectrometry
}

\author{
CRISTIANO RIZZO, SARA BOENZI, RONALD J.A. WANDERS, MARINUS DURAN, \\ UBALDO CARUSO, AND CARLO DIONISI-VICI \\ Division of Metabolism [C.R., S.B., C.D.-V.], Bambino Gesù Research Institute, 00165 Rome, Italy; \\ Academic Medical Center Amsterdam [R.J.A.W, M.D.], Department of Pediatrics \& Clinical Chemistry, \\ Laboratory of Genetic Metabolic Diseases, $1105 A Z$ Amsterdam, The Netherlands; and Department of \\ Pediatrics [U.C.], Laboratory for the Study of Inherited Metabolic Diseases, Research Institute G. Gaslini, \\ 16148 Genoa, Italy.
}

\begin{abstract}
ABST
Patients with inherited defects of peroxisomal metabolism, a
class of diseases with marked clinical and genetic heterogeneity,
show a characteristic phenotype in most cases with severe neu-
rologic impairment, craniofacial abnormalities, and hepatic and
kidney dysfunction. For the differential diagnosis of clinically
suspected cases, a complex biochemical and genetic approach is
required. Analysis of plasma very-long-chain fatty acids is a
reliable screening method to detect most but not all peroxisomal
disorders. To study the potential presence of abnormal acylcar-
nitine species in plasma and blood, we screened by tandem mass
spectrometry a series of patients affected by a peroxisome bio-
genesis disorder (PBD) and compared the results with those
obtained in patients with isolated peroxisomal defects (e.g. D-
bifunctional protein deficiency, X-linked adrenoleukodystrophy)
and mitochondrial long-chain fatty acid oxidation defects. The
most relevant finding observed in plasma of patients with PBD
was a significant increase of long-chain dicarboxylic C16- and
C18-carnitine, i.e. hexadecanedioyl- and octadecanedioyl-
carnitine, with high dicarboxylycarnitine/monocarboxylylcarni-
\end{abstract}
Peroxisomes are cellular organelles that contribute to several metabolic pathways, most of which are related to the metabolism of lipids (1). The inherited defects that affect peroxisome metabolism, called peroxisomal disorders, are clinically and genetically heterogeneous and can be roughly classified into two main groups, the peroxisome biogenesis disorders (PBD) and single peroxisomal enzyme deficiencies. The PBD group includes Zellweger syndrome, neonatal adrenoleukodystrophy,

Received March 22, 2002; accepted November 18, 2002.

Correspondence: Carlo Dionisi-Vici, Division of Metabolism, Bambino Gesù Research Institute, Piazza S. Onofrio 4, 00165 Rome, Italy; e-mail: dionisi@opbg.net

This work was supported by grant $00 / 01 / 080$ of the Italian Ministry of Health (C.D.-V.).

DOI: $10.1203 / 01 . P D R .0000064902 .59052 .0 F$ tine ratio. Elevation of very long-chain acylcarnitines $\mathrm{C} 24-$ and C26-, i.e. lignoceroyl- and cerotoyl-carnitine, was detected in some PBDs and in D-bifunctional protein deficiency. Similar abnormalities were also found in neonatal screening blood spots. Detection of these compounds alone, in the absence of other shorter-chain acylcarnitines, is highly specific and characteristic of PBD, as confirmed by the differing profiles observed in patients with adrenoleukodystrophy and mitochondrial longchain fatty acid oxidation defects. Our study adds a novel method to the diagnosis of PBD, which may also be of benefit for future neonatal mass screening programs based on acylcarnitine profiling. (Pediatr Res 53: 1013-1018, 2003)

\section{Abbreviations:}

PBD, peroxisome biogenesis disorders

TMS, tandem mass spectrometry

GC/MS, gas chromatography/mass spectrometry

VLCFA, very-long-chain fatty acids

CACT, carnitine acyl-carnitine translocase infantile Refsum disease, and rhizomelic chondrodysplasia punctata type 1 , as the most frequent phenotypes and have so far been associated with 16 different complementation groups (2). Clinically, neonatal adrenoleukodystrophy and infantile Refsum disease are phenotypic variants of the Zellweger spectrum (3), whereas rhizomelic chondrodysplasia punctata type 1 represents a well-defined entity caused in most cases by mutations in the PEX7 gene (4). Interestingly, the phenotype of patients affected by isolated enzyme defects (acyl-CoA oxidase deficiency, D-bifunctional protein deficiency, and thiolase deficiency) share many similarities with patients with PBD (4).

Given the marked heterogeneity in the phenotypic expression of patients, the biochemical diagnosis of peroxisomal 
disorders is complex. Analysis of plasma very-long-chain fatty acids (VLCFA) is a reliable screening method to detect most but not all peroxisomal disorders (5). If VLCFAs are found to be abnormal, then the flowchart for the differential diagnosis of peroxisomal disorders requires further studies in blood (plasmalogen, bile acids, phytanic/pristanic acid determinations) and fibroblasts (i.e. de novo plasmalogen biosynthesis, DHAPAT (dihydroxyacetonephosphate acyltransferase) activity, fatty acid $\alpha$ - and $\beta$-oxidation, immunoblot analysis, peroxisome morphology, complementation studies and molecular analyses) (5).

Here we report on the characteristic abnormalities of circulating acylcarnitines observed in patients with PBD as detected by tandem mass spectrometry (TMS). Our study adds a novel method to the diagnosis of PBD, which may also be of relevance for future neonatal mass screening programs.

\section{METHODS}

\section{Patients}

Plasma and/or neonatal screening blood spots for acylcarnitine analysis was obtained from 10 patients with a proven PBD. All showed phenotypic features characteristic of the Zellweger spectrum, including severe neurologic impairment, craniofacial abnormalities, and hepatic and kidney dysfunction. Biochemically, they presented with elevated plasma levels of VLCFA, phytanic and pristanic acids, abnormal bile acid profile, low plasmalogens in erythrocytes, and dicarboxylicand epoxydicarboxylic-aciduria (Table 1). Diagnosis of PBD was confirmed by fibroblast studies.

For comparison, we analyzed samples from patients with isolated peroxisomal defects (D-bifunctional protein deficiency and X-linked adrenoleukodystrophy) and mitochondrial longchain fatty acid oxidation defects [long-chain 3-hydroxyacylCoA dehydrogenase, very-long-chain acyl-CoA dehydrogenase, carnitine acyl-carnitine translocase (CACT)]. For the latter category, samples corresponded to acute phases of metabolic decompensation.

\section{MATERIALS AND METHODS}

The internal acylcarnitine standards were $\left[{ }^{2} \mathrm{H}_{3}\right]$ freecarnitine, $\left[{ }^{2} \mathrm{H}_{3}\right]-\mathrm{C}_{2}$ (acetylcarnitine), $\left[{ }^{2} \mathrm{H}_{3}\right]-\mathrm{C}_{3}$ (propionylcarnitine), $\left[{ }^{2} \mathrm{H}_{3}\right]-\mathrm{C}_{4}$ (butyrylcarnitine), $\left[{ }^{2} \mathrm{H}_{9}\right]$ iso- $\mathrm{C}_{5}$ (isovalerylcarnitine), $\left[\begin{array}{lllll}{ }^{2} \mathrm{H}_{3} & \mathrm{C}_{8} & \text { (octanoylcarnitine), } & {\left[{ }^{2} \mathrm{H}_{9}\right.} & \mathrm{C}_{14}\end{array}\right.$ (myristoylcarnitine), and $\left[{ }^{2} \mathrm{H}_{3}\right] \mathrm{C}_{16}$ (palmitoylcarnitine; Cambridge Isotopes Laboratories, Andover, MA, U.S.A.). Acylcarnitines were analyzed from $10 \mu \mathrm{L}$ of plasma or from filter paper (W303) blood spot (equivalent to $10 \mu \mathrm{L}$ of whole blood) as butyl esters. Briefly, the blood was extracted with $400 \mu \mathrm{L}$ of a solution containing labeled internal standard dissolved in methanol and evaporated under nitrogen stream. Eighty microliters of butanolic- $\mathrm{HCl}$ (Regis Technologies, Morton Grove, IL, U.S.A.) was added to each sample, heated at $65^{\circ} \mathrm{C}$ for 20 min, and evaporated. The sample was then reconstituted with $200 \mu \mathrm{L}$ of water/acetonitrile/formic acid (50/50/0.025). The butylated samples were analyzed with a triple quadruple tandem mass spectrometer (Sciex API 365; PE Sciex Instrument, Concord, ON, Canada). The TMS method was the positive precursor ion scan of $\mathrm{m} / \mathrm{z} 85$, the characteristic fragment of acylcarnitine butyl ester derivatives, from 200 to $650 \mathrm{Da}$ (6). The collision energy was $44 \mathrm{eV}$.

The ion intensities of the various acylcarnitines were compared with those of corresponding internal standards by computer analysis (Neonatal Script, PE Sciex). For the long-chain analytes lacking specific internal standards, results were expressed as signal intensity ratios to that of the internal standard $\left[{ }^{2} \mathrm{H}_{3}\right] \mathrm{C}_{16}$ (palmitoylcarnitine). Reference values (97.5 percentile) were obtained from 130 healthy age-matched controls.

Owing to the small size of the sample of patients, the nonparametric Mann-Whitney $U$ test for independent samples was used to analyze possible differences between patients and controls. The level of significance was set at $p<0.05$.

To confirm the identification of acylcarnitine species detected by TMS experiments, we performed gas chromatography/mass spectrometry (GC/MS) analysis of organic acids constituting the acylcarnitines by selected ion monitoring accordingly to a previously described method (7). For obtaining

Table 1. Plasma levels of VLCFA (C26, C26/C22), phytanic acid, pristanic acid, and bile acid intermediates in patients with peroxisomal biogenesis disorders, and D-bifunctional protein deficiency.

\begin{tabular}{|c|c|c|c|c|c|c|}
\hline & $\mathrm{C} 26$ & $\mathrm{C} 26 / \mathrm{C} 22$ & $\begin{array}{l}\text { Phytanic } \\
\text { acid }\end{array}$ & $\begin{array}{l}\text { Pristanic } \\
\text { acid }\end{array}$ & THCA & DHCA \\
\hline \multicolumn{7}{|l|}{ Peroxisomal biogenesis disorders } \\
\hline Patient 1 & 12.4 & 0.98 & 0.8 & 0.1 & & \\
\hline Patient 3 & 5.6 & 0.62 & 15.0 & 6.3 & & \\
\hline Patient 4 & 3.9 & 0.43 & 32.6 & 12.8 & & \\
\hline Patient 5 & 2.7 & 0.16 & & & 9.0 & 44.0 \\
\hline Patient 8 & 10.7 & 0.78 & $<0.1$ & $<0.1$ & 56.8 & 88.1 \\
\hline Patient 9 & 9.5 & 0.30 & 15.8 & 2.4 & 31.8 & 7.7 \\
\hline Patient 10 & 8.8 & 0.49 & 11.7 & 7.2 & & \\
\hline D-bifunctional protein deficiency & 5.4 & 0.14 & & & 13.4 & 25.4 \\
\hline Controls & $0.45-1.32$ & $0.00-0.02$ & $<10$ & $<3$ & $<0.1$ & $<0.1$ \\
\hline
\end{tabular}

THCA, trihydroxycholestanoic acid; DHCA, dihydroxycholestanoic acid.

Values are expressed as $\mu \mathrm{mol} / \mathrm{l}$. Abnormal values in bold. 
total fatty acids, previous extraction, and derivatization, plasma was first hydrolyzed with $1 \mathrm{M} \mathrm{NaOH}$ for $1 \mathrm{~h}$ at $100 \mathrm{C}^{\circ}$. Internal standards were the commercially available chemicals tetradecanedioic acid (C14 dicarboxylic), hexadecanedioic acid (C16 dicarboxylic), tetracosanoic (lignoceric) acid (C24), and hexacosanoic (cerotic) acid (C26; Sigma Chemical Co., St. Louis, MO, U.S.A.). This study was approved by Institutional Review Board, and informed consent was obtained from patients' families.

\section{RESULTS}

Plasma long-chain dicarboxylic acylcarnitines and very long-chain acylcarnitines were picked up by tandem mass spectrometry (Fig. 1). As shown in Table 2, plasma acylcarnitine analysis showed that long-chain dicarboxylic C16- (hexadecanedioyl-) and dicarboxylic C18- (octadecanedioyl-) carnitine esters were increased in patients with PBD. The results of the analyses in neonatal screening blood spots in four of 10 patients with PBD showed a similar pattern, with less pronounced abnormalities than in plasma. However, in all blood samples, at least one of the abnormal acylcarnitine esters was above the control range. Statistical evaluation showed that by comparing levels of these acylcarnitine species in patients and controls, patients with PBD had significantly higher levels than controls, both in plasma and in blood samples (Table 2).

In addition to these abnormalities, two other compounds were detected in some patients with PBD. According to the molecular weight using the precursor ion of mass $85 \mathrm{~m} / \mathrm{z}$, they could correspond to dicarboxylyl C18:1- or monocarboxylyl C24-carnitine (mass 568) and to dicarboxylyl C20:1- or monocarboxylyl C26-carnitine (mass 596). To resolve this question, we conducted additional experiments using hydrolysis and GC/MS analysis, which demonstrated the presence of C24(lignoceric) and C26- (cerotic) fatty acids but not C18:1 and C20:1 fatty acids as constituents (Table 2).

Different from PBDs, in plasma and blood from a Dbifunctional protein-deficient patients, we found a major increase of lignoceroyl- and cerotoyl-carnitine (Table 1). In all three patients with X-linked adrenoleukodystrophy, plasma acylcarnitine profiles were completely normal.

Patients with mitochondrial defects of long-chain fatty acid $\beta$-oxidation (i.e. long-chain 3-hydroxyacyl-CoA dehydrogenase and very-long-chain acyl-CoA dehydrogenase deficiencies) showed their respective characteristic acylcarnitine spe-

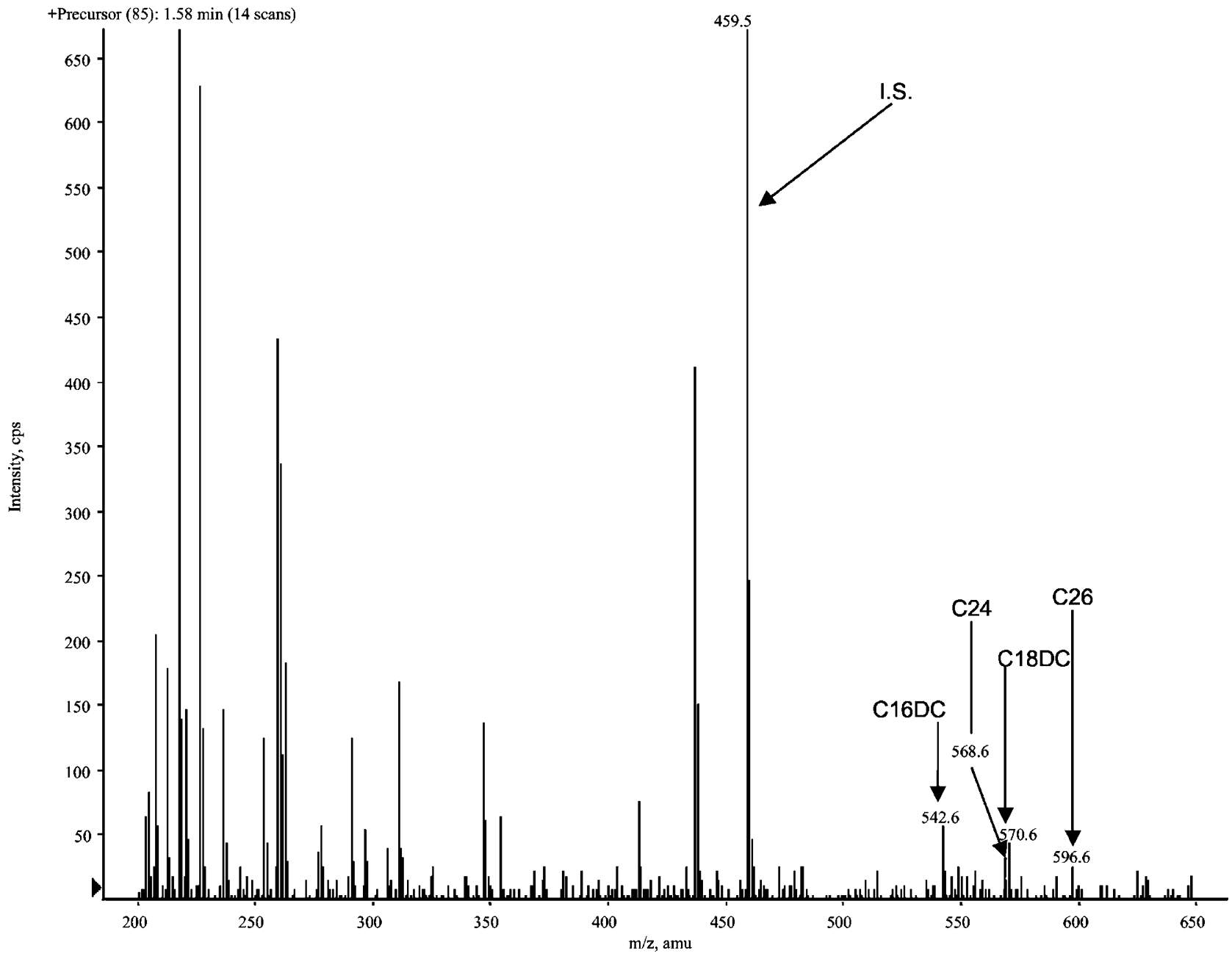

Figure 1. Plasma acylcarnitine profile obtained by TMS analysis scanning for precursor ions of $\mathrm{m} / \mathrm{z} 85$ from a patient with a PBD (Zellweger syndrome). The masses are as follows: internal standard $\mathrm{d}_{3}$ palmitoyl carnitine $\left(\mathrm{C} 16, \mathrm{M}^{+} 459.5\right)$, exadecanedioyl-carnitine $\left(\mathrm{C} 16 \mathrm{DC}, \mathrm{M}^{+}\right.$542.6), octadecanedioyl-carnitine (C18DC, $\mathrm{M}^{+}$570.6), lignoceroyl-carnitine (C24, $\mathrm{M}^{+}$568.6), cerotoyl-carnitine (C26, $\mathrm{M}^{+}$596.6) 
Table 2. Acylcarnitines in plasma and blood samples from patients with peroxisomal biogenesis disorders, D-bifunctional protein deficiency, X-linked adrenoleucodystrophy, patients with fatty acid oxidation defects and in controls $(n=103)$

\begin{tabular}{|c|c|c|c|c|c|c|c|c|}
\hline & \multicolumn{4}{|c|}{ Plasma } & \multicolumn{4}{|c|}{ Blood } \\
\hline & C16-DC & C18-DC & $\mathrm{C} 24$ & $\mathrm{C} 26$ & C16-DC & C18-DC & $\mathrm{C} 24$ & $\mathrm{C} 26$ \\
\hline \multicolumn{9}{|l|}{ Peroxisomal biogenesis disorders } \\
\hline Patient 2 & 0.15 & 0.35 & 0.04 & 0.04 & 0.03 & 0.08 & 0.11 & 0.11 \\
\hline Patient 3 & 0.15 & 0.11 & 0.05 & 0.01 & & & & \\
\hline Patient 4 & 0.12 & 0.07 & 0.01 & 0.01 & 0.18 & 0.01 & 0.03 & 0.07 \\
\hline Patient 5 & 0.16 & 0.06 & 0.01 & 0.06 & & & & \\
\hline Patient 7 & 0.18 & 0.05 & 0.04 & 0.03 & & & & \\
\hline Patient 8 & 0.08 & 0.01 & 0.02 & 0.04 & & & & \\
\hline Patient 9 & 0.06 & 0.05 & 0 & 0.02 & & & & \\
\hline Patient 10 & & & & & 0.18 & 0.11 & 0.07 & 0.09 \\
\hline Median & $0.15 *$ & $0.07 *$ & 0.04 & 0.04 & $0.18 \dagger$ & $0.09 \dagger$ & 0.09 & 0.08 \\
\hline D-bifunctional protein deficiency & 0.02 & 0.01 & 0.05 & 0.07 & 0.09 & 0.03 & 0.16 & 0.36 \\
\hline LCHAD (patient 1) & 0.03 & 0.02 & & & 0.01 & 0.01 & 0.01 & 0.01 \\
\hline LCHAD (patient 2) & 0.02 & 0.01 & & & 0.02 & 0.04 & 0.03 & 0.01 \\
\hline CACT & & & & & 0.23 & 0.11 & 0.13 & 0.01 \\
\hline VLCAD & & & & & 0.02 & 0.01 & 0.02 & 0.01 \\
\hline \multicolumn{9}{|l|}{ Controls } \\
\hline Upper Thresholds & 0.03 & 0.02 & 0.04 & 0.04 & 0.07 & 0.05 & 0.09 & 0.09 \\
\hline Median & 0.007 & 0.003 & 0.007 & $<0.001$ & $<0.001$ & $<0.001$ & 0.02 & 0.01 \\
\hline
\end{tabular}

LCHAD, long-chain 3-hydroxyacyl-CoA dehydrogenase; VLCAD, very-long chain acyl-CoA dehydrogenase. long-chain acylcarnitines: C16-DC, hexadecanedioyl-carnitine; C18-DC, octadecanedioyl-carnitine. Very long-chain acylcarnitines: C24, lignoceroyl-carnitine; C26, cerotoyl-carnitine. Values expressed as arbitrary unit based on signal response ratio to trideuterated C16 internal standard. In bold abnormal values. $* p<0.001, \dagger p<0.003$.

cies (8) but none of those observed in PBD. Interestingly, in CACT deficiency in addition to typical $\mathrm{C} 16$ and $\mathrm{C} 18$ monocarboxylic carnitine esters, the corresponding dicarboxylic C16- and C18-acylcarnitines were also elevated together with raised lignoceroyl-carnitine (Table 2).

Moreover, the ratio between long-chain dicarboxylyl- and its corresponding monocarboxylyl-carnitine ester was markedly higher in PBDs when compared with mitochondrial long-chain fatty acid oxidation disorders. The ratio between hexadecanedioyl- and hexadecanoyl-carnitine was 1.5 and 0.23 in PBDs versus mitochondrial defects in plasma, and 0.12 versus 0.02 in blood, respectively. The ratio between octadecanedioyl- and octadecanoyl-carnitine was 1.4 and 0.16 in PBDs versus mitochondrial defects in plasma, and 0.11 versus 0.04 in blood (median values).

Because of the lack of commercially available deuterated acylcarnitine standards for TMS analysis, GC/MS experiments were necessary to confirm the elevation of acylcarnitine compounds after alkaline hydrolysis (Fig. 2). The identification of octadecanedioic acid was based on the M-15 fragment (443), on the ions m/z 204 and 217 characteristic of long-chain dicarboxylic TMS derivatives (9), and on the retention time at 43 and $48 \mathrm{~min}$, respectively.

\section{DISCUSSION}

Our study demonstrates the presence of a peculiar profile of circulating acylcarnitines in patients with PBD. The most characteristic compounds detectable both in plasma and in blood were long-chain dicarboxylic acylcarnitines with a straight carbon chain length of 16 and 18 atoms (i.e. hexadecanedioyl- and octadecanedioyl-carnitine). In addition, some PBD samples were characterized by a very long-chain acylcarnitinemia (C24-C26 acylcarnitines).

In the single patient with D-bifunctional protein deficiency, the profile of circulating acylcarnitines was different compared with PBDs, with elevation limited to very long-chain acylcarnitines. No acylcarnitine abnormalities were detectable in patients with X-linked adrenoleukodystrophy.

Consistent with a previous report on the increased excretion of long-chain dicarboxylic acids, including $\mathrm{C} 16$, in patients with Zellweger syndrome and neonatal adrenoleukodystrophy (10), the mechanism behind the accumulation of dicarboxylic acylcarnitines is probably related to the microsomal generation of dicarboxylic acids. Under all conditions, there is some $\omega$-oxidation of long-chain fatty acids, including C16:0 and C18:0, that produces the corresponding dicarboxylic acids, followed by activation by a microsomal dicarboxylyl-CoA synthetase (11). The activated CoA esters then undergo some cycles of $\beta$-oxidation in the peroxisome up to a certain point, probably C10-dicarboxylyl-CoA, which is then transported to the mitochondria in the form of its carnitine ester (Fig. 3). This explains the abnormalities observed in PBDs. The normal level of long-chain dicarboxylyl-carnitines indicates that this pathway is functionally normal in D-bifunctional protein deficiency 


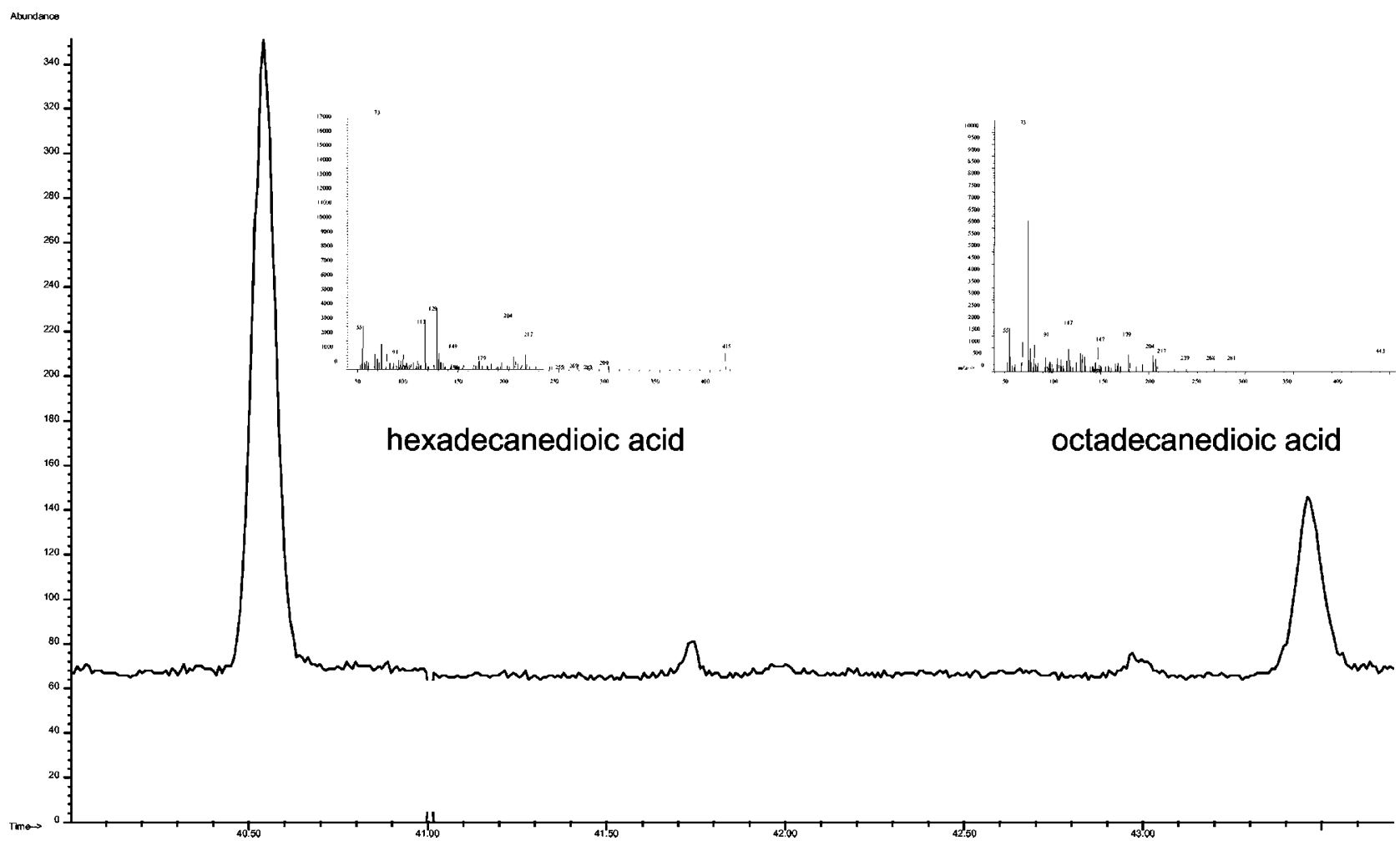

Figure 2. Plasma GC/MS analysis of total fatty acids (detail) in a patient with a PBD (Zellweger syndrome) after alkaline hydrolysis showing two peaks, and their respective mass spectra, corresponding to hexadecanedioic acid (C16 dicarboxylic) and to octadecanedioic acid (C18 dicarboxylic).

as well as in X-linked adrenoleukodystrophy. This may suggest that L-bifunctional protein rather than D-bifunctional protein is involved in dicarboxylic acid $\beta$-oxidation.

The elevation of very-long-chain acylcarnitines observed in some PBDs (probably those with functionally normal membrane activation/transport of VLCFA) and in D-bifunctional protein deficiency is likely to be due to the impaired oxidation of VLCFA after their activation to CoA esters by very-longchain acyl-CoA synthetase, an enzyme located on peroxisomal membrane. Accumulation of CoA esters in the peroxisomal matrix could therefore activate their conversion to carnitine esters at this subcellular level (Fig. 3). Conversely, the finding of a normal acylcarnitinemia in X-linked adrenoleukodystrophy (a disease caused by a defect in a peroxisome membrane protein) could be due to the impaired transport/activation of very-long-chain fatty acyl-CoAs across the peroxisomal membrane without an increase in the matrix. Similarly, in some PBDs (probably those fully lacking membrane-associated proteins), the impaired activation of VLCFA through very-longchain acyl-CoA synthetase does not allow the transesterification to carnitine esters.

Among mitochondrial long-chain fatty acid oxidation disorders, only CACT deficiency shared some similarities with PBD. In this transport defect of long-chain fatty acids across the mitochondrial membrane, the profile of plasma acylcarnitines showed, in addition to the characteristic monocarboxylic long-chain acylcarnitines (8), the elevation of their corresponding dicarboxylic esters of 16 and 18 carbon atoms. However, careful evaluation of whole acylcarnitine spectrum allows a clear distinction between mitochondrial and peroxisomal disorders.
In CACT deficiency, the excess of long-chain fatty acids in the cytosol are first subject to $\omega$-oxidation and the C16- and C18-dicarboxylyl-CoA esters then undergo some $\beta$-oxidation in peroxisomes but only to a limited extent, because the final product, i.e. C10-dicarboxylyl-CoA, cannot enter the mitochondrion since the impaired transport of C10-dicarboxylyl-carnitine.

Remarkably, in contrast with mitochondrial disorders, in PBDs, the long-chain acylcarnitinemia was characterized by a higher dicarboxylyl- on monocarboxylyl-carnitine esters ratio. This seems to indicate that in PBDs, the peroxisomal $\beta$-oxidation chain shortening of long-chain dicarboxylyl-CoAs is primarily deficient (12), whereas long-chain monocarboxylic acids are normally oxidized in the mitochondria. On the contrary, in mitochondrial disorders, the primary defect is related to the impaired oxidation of long-chain monocarboxylic acids, and the accumulation of dicarboxylic acyl-CoA or carnitine esters occurs only as a secondary event.

In conclusion, long-chain acylcarnitinemia with high dicarboxylycarnitine on monocarboxylylcarnitine ratio and verylong-chain acylcarnitinemia represent two emerging opportunities to identify inherited peroxisomal disorders and to differentiate them from mitochondrial fatty acid oxidation defects. Our finding represents an important extension of TMS and offers a novel marker for screening diagnosis of PBD. The recent use of TMS has opened up a "new era" in this field, expanding the range of diseases detectable at birth through neonatal mass screening to $>30$ different defects, including aminoacidopathies, defects of fatty acid oxidation, organic acidurias, and some disorders of the urea cycle (13). Although, at present, PBDs represent a class of untreatable diseases that 


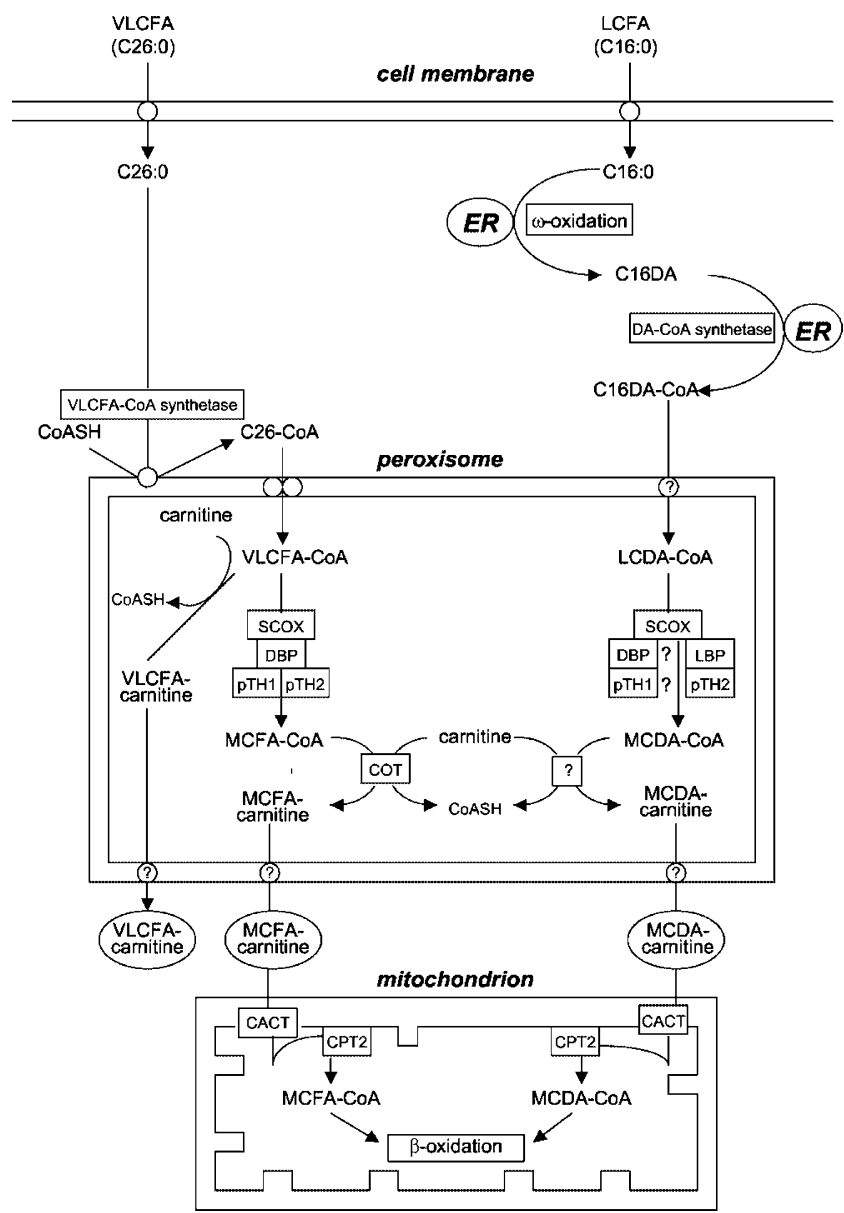

Figure 3. Schematic representation of the fatty acid $\beta$-oxidation machinery catalyzing the oxidation of VLCFA and of long-chain dicarboxylic acids (LCDA) in the peroxisome and the oxidation of long-chain fatty acids (LCFA) and of medium-chain dicarboxylic acids (MCDA) in the mitochondrion. ER, endoplasmic reticulum; SCOX, straight-chain acyl-CoA oxidase; DBP, Dbifunctional protein; LBP, L-bifunctional protein; pTH, peroxisomal 3-ketoacyl-CoA thiolase; COT, carnitine octanoyltransferase; CPT2, carnitine palmitoyltransferase 2 .

do not fulfill the criteria for neonatal mass screening, acylcarnitine analysis may be very helpful, similar to TMS evaluation of bile acid intermediates and VLCFA $(14,15)$, in cases in which the index patient has died without any biochemical analyses being done and the suspicion of a PBD has only come up much later.

Interestingly, the comparative evaluation of acylcarnitine analysis with the most traditionally used GC/MS methods for the diagnosis of peroxisomal disorders demonstrated that VLCFA and bile acids intermediates allowed the detection of all PBDs and D-bifunctional protein deficiency, whereas plasma phytanic and pristanic acids were elevated only in $62 \%$ and $50 \%$ of cases, respectively (Table 1). However, this latter finding is not surprising as it is already known that the elevation of these metabolites depends on the age of subjects as well as on dietary intake (16).

Two relevant technical points concern the use of TMS. Currently, methods for neonatal mass screening use for the precursor ion mass 85 a window between 200 and $500 \mathrm{Da}$ (6), a range that does not allow the detection of very-long- and long-chain acylcarnitines. Furthermore, to increase both fragmentation and sensitivity for dicarboxylic acylcarnitines, we have applied a higher collision energy. We therefore recommend widening the mass window range up to $650 \mathrm{Da}$ and using a collision energy of $44 \mathrm{eV}$ to identify the characteristic compounds of PBD.

\section{REFERENCES}

1. Wanders RJ, Vreken P, Ferdinandusse S, Jansen GA, Waterham HR, van Roermund $\mathrm{CW}$, Van Grunsven EG 2001 Peroxisomal fatty acid alpha- and beta-oxidation in humans: enzymology, peroxisomal metabolite transporters and peroxisomal diseases. Biochem Soc Trans 29:250-267

2. Gould SJ, Valle D 2000 Peroxisome biogenesis disorders genetics and cell biology. Trends Genet 16:340-345

3. Gould SJ, Raymond GV, Valle D 2001 The peroxisome biogenesis disorders. In: Scriver CR, Beaudet AL, Sly WS, Valle D (eds) The Metabolic and Molecular Bases of Inherited Disease. 8th ed. McGraw-Hill, New York, NY, pp 3181-3217

4. Motley AM, Hettema EH, Hogenhout EM, Brites P, ten Asbroek AL, Wijburg FA, Baas F, Heijmans HS, Tabak HF, Wanders RJ, Distel B 1997 Rhizomelic chondrodysplasia punctata is a peroxisomal protein targeting disease caused by a nonfunctional PTS2 receptor. Nat Genet 15:377-380

5. Wanders RJA, Barth PG, Heymans HSA 2001 Single peroxisomal disorders enzyme deficiencies. In: Scriver CR, Beaudet AL, Sly WS, Valle D (eds) The Metabolic and Molecular Bases of Inherited Disease. 8th ed. McGraw-Hill, New York, NY, pp 3219-3256

6. Rashed MS, Bucknall MP, Little D, Awad A, Jacob M, Alamoudi M, Alwattar M, Ozand PT 1997 Screening blood spots for inborn errors of metabolism by electrospray tandem mass spectrometry with a microplate batch process and a computer algorithm for automated flagging of abnormal profiles. Clin Chem 43:1129-1141

7. Rizzo C, Bertini E, Piemonte F, Leuzzi V, Sabetta G, Federici G, Luchetti A, Dionisi-Vici C 2000 Oxidative abnormalities in Menkes disease. J Inherit Metab Dis 23:349-351

8. Vreken P, Van Lint AEM, Bootsma AH, Overmars H, Wanders RJA, Van Gennip H 1999 Quantitative plasma acylcarnitine analysis using electrospray tandem mass spectrometry for the diagnosis of organic acidemias and fatty acid oxidation defects. J Inherit Metab Dis 22:302-306

9. Chalmers RA, Lawson AM 1982 Organic Acids in Man. Analytical Chemistry, Biochemistry and Diagnosis of the Organic Acidurias. London, Chapman \& Hall, p 119

10. Rocchicioli F, Aubourg P, Bougneres PF 1996 Medium- and long-chain dicarboxylic aciduria in patients with Zellweger syndrome and neonatal adrenoleukodystrophy. Pediatr Res 20:62-66

11. Vameq J, de Hoffmann E, Van Hoof F 1985 The microsomal dicarboxyl-CoA synthetase. Biochem J 230:683-693

12. Reddy JK, Hashimoto T 2001 Peroxisomal $\beta$-oxidation and peroxisome proliferatoractivated receptor $\alpha$ : an adaptive metabolic system. Annu Rev Nutr 21:193-230

13. Levy HL 1998 Newborn screening by tandem mass spectrometry: a new era. Clin Chem 44:2401-2402

14. Bootsma AH, Overmars H, van Rooij A, van Lint AE, Wanders RJ, van Gennip AH, Vreken P 1999 Rapid analysis of conjugated bile acids in plasma using electrospray tandem mass spectrometry: application for selective screening of peroxisomal disorders. J Inherit Metab Dis 22:307-310

15. Johnson DW 2000 A rapid screening procedure for the diagnosis of peroxisomal disorders: quantification of very long-chain fatty acids, as dimethylaminoethyl esters, in plasma and blood spots, by electrospray tandem mass spectrometry. J Inherit Metab Dis 23:475-486

16. Verhoeven NM, Wanders RJA, Poll-The BT, Saudubray JM, Jakobs C 1998 The metabolism of phytanic and pristanic acid in man: a review. J Inherit Metab Dis 21:697-728 